\title{
A purely central movement aftereffect induced by binocular viewing of dynamic visual noise
}

\author{
YEHOSHUA Y. ZEEVI \\ Massachusetts Institute of Technology, Cambridge, Massachusetts \\ and \\ GEORGE A. GERI \\ University of Dayton Research Institute, Williams AFB, Arizona
}

\begin{abstract}
Observers viewed dynamic visual noise binocularly with a neutral density filter placed over the right eye. Twelve of 13 observers perceived depth and coherent motion of two counterdirectional distributions of dot-planes. Three of the 12 observers saw one plane as much more distinct than the others; thus, for those observers, that dot-plane could be used as a form-free central movement stimulus. Sudden removal of the neutral density filter resulted in a short-lived movement aftereffect (MAE). The magnitude of the purely central MAE, unlike those of classical MAEs, was independent of both the magnitude and the time spent viewing the inducing movement. These results suggest a distinction between the purely central MAE reported here and the central (binocular) MAEs induced by stimulus movement on the retina.
\end{abstract}

The dynamic visual noise (DVN) stereophenomenon is the perception of depth and coherent motion that results if an interocular intensity difference is introduced during the binocular viewing of DVN (Falk \& Williams, 1980; Ross, 1974; Tyler, 1974, 1977). The binocular disparity resulting from the intensity difference transforms the random pattern of dots of the DVN into a distribution of coherently moving dot-planes which appear separated in depth. It has been noted that under certain stimulus conditions one of the moving dot-planes appears most distinct (Falk \& Williams, 1980), and hence that plane can be used as a visual stimulus independently of the other planes. For example, Zeevi and Medina (1984) showed that the perceived velocity associated with this single dotplane could serve as a stimulus for smooth eye movements.

Classical movement aftereffects (MAEs), such as the waterfall illusion, are induced by stimulus movement on the retina, and thus may be classified as peripheral MAEs. Another type of MAE results if one eye is stimulated by a moving stimulus while the MAE is observed by the other eye (cf. Anstis \& Moulden, 1970; Barlow \& Brindley, 1963; Favreau, 1976). In this case, too, movement information exists at the retinal level, although the process of intraocular transfer indicates central involvement in the production of the MAE. A third type of MAE, here referred to as a purely central MAE, is produced by perceived movement which does not exist at the retinal level.

This research was supported in part by Air Force Contract No. F33615-81-K-0011. Y. Zeevi's present address is: Department of Electrical Engineering, Technion, Haifa, Israel 32000. All correspondence should be addressed to G. Geri, UDRI, P.O. Box 44, Higley, AZ 85236.
For instance, Papert (1964) used dynamically generated random-element stereograms which appeared as moving depth contours when viewed binocularly and as a random dot array when viewed with either eye alone. These dynamic stereograms induced an MAE that was shorter for a given inducing time and was more sporadic and less pronounced than peripheral or binocular MAEs. Subsequent research on the central MAE has resulted in seeming contradiction. Anstis (1980; Anstis \& Moulden, 1970) published descriptions of the central MAE that are similar to those of Papert. On the other hand, Fox, Patterson, and Lehmkuhle (1982) used moving cyclopean contours to induce purely central MAEs which lasted as long as $20 \mathrm{sec}$ and which those authors described as qualitatively similar to peripheral and binocular MAEs.

In the present study we further investigated the unique properties of the purely central MAE. We used as a central movement stimulus the DVN stereophenomenon. This stimulus is preferable to those used by Papert (1964) and Fox et al. (1982), in that it contains no global form or edge information. It was our primary purpose to obtain quantitative data on the relationship between the magnitude of a central inducing movement and the magnitude of the purely central MAE it produces.

\section{METHOD}

\section{Observers}

Data were obtained from 3 emmetropic male observers, J.H., D.W., and G.G., who were 21,22 , and 32 years of age, respectively. The 3 observers were chosen based on their perceiving one moving dot-plane to be much more distinct than the others. The appearance of the aftereffect was confirmed by 9 other observers; 1 additional observer failed to perceive movement or depth in the DVN display. 


\section{Apparatus}

Various methods for generating DVN have been described (Falk \& Williams, 1980; MacDonald, 1977; Mezrich \& Rose, 1977; Neill, 1981; Ross, 1974). We found that a detuned television receiver (cf. Tyler, 1974), which displayed pixels of high density and random rather than uniformly high contrast, produced DVN which most consistently resulted in an MAE. We used a Sony Trinitron Monitor (Model CVM-1225) with its screen masked to give a $15^{\circ}$ (horizontal) $\times 11^{\circ}$ (vertical) rectangular field at a viewing distance of $0.8 \mathrm{~m}$. The observers viewed the DVN stimulus through a large beamsplitter (see Figure 1) that allowed either a moving spot or a red LED to be binocularly superimposed on the DVN display. The moving spot was produced on an oscilloscope by a function generator and its velocity was controlled by the observer. The LED was mounted on a carriage which could be moved along a graduated track. The mean luminance of the DVN display as viewed through the beamsplitter was $28 \mathrm{fL}$. An interocular luminance difference was produced by neutral density filters placed in front of the right eye of each observer. While viewing the inducing stimuli, the observers were asked to fixate a small black spot placed on the TV monitor screen. No fixation point was present during the LED depth measurements. Chin- and headrests were provided.

\section{Procedure}

Three observers participated in the study. After 4-5 min of adaptation to the ambient illumination and the dynamic noise produced by the TV monitor, a neutral density filter was placed before each observer's right eye. The observer first set the depth of the superimposed moving spot to be coplanar with the most distinct dot-plane (i.e., the plane used as the movement stimulus). While fixating the center of the DVN display, the observer adjusted the velocity of the moving spot to match that of the moving dot-plane. A total of eight settings were made for each interocular luminance difference over the course of four experimental sessions. Within each session, seven luminance differences corresponding to neutral densities of $1.0,1.3,1.5,1.7,2.0,2.3$, and 2.5 were presented randomly. The MAE was induced by allowing the observer to view the coherent motion of the recessed plane for $20 \mathrm{sec}$ and then suddenly removing the attenuating filter. The observer then attempted to match the velocity of the moving spot, which was now set coplanar with the

\section{DVN DISPLAY}

or

BACK-ILLUMINATED GROUND GLASS

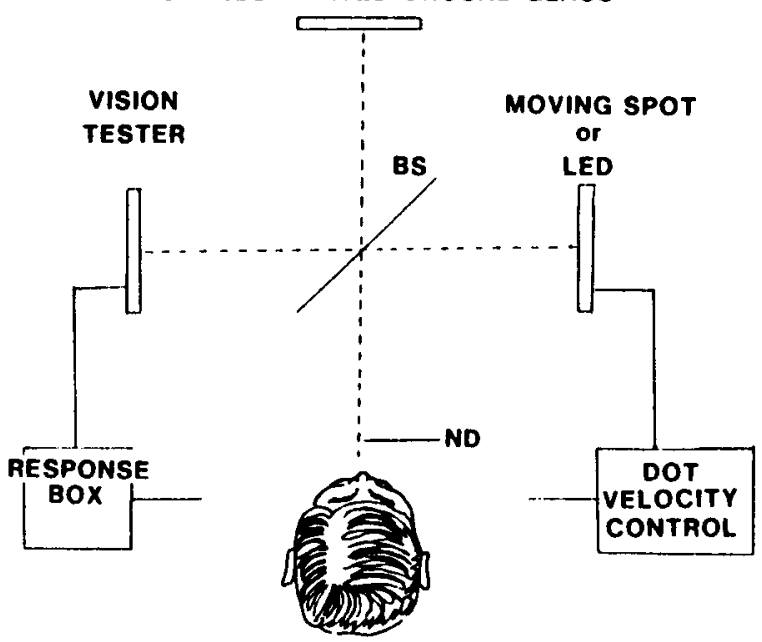

Figure 1. A diagram of the apparatus used for the movement aftereffect and depth measurements and the calibration of the DVN display. The beamsplitter (BS) could be rotated $90^{\circ}$ and the neutral density filters (ND) were removed as required.
DVN display, to that of the resulting aftereffect. Because the aftereffect lasted only a few seconds, several trials were needed for each setting, with the observer making adjustments so that the velocity of the aftereffect was gradually approached. Four settings were obtained for each interocular luminance difference over the four experimental sessions.

It is well established that eye movements affect the perceived velocity associated with the DVN stereophenomenon (Tyler, 1974; Ward \& Morgan, 1978; Zeevi \& Medina, 1984). We therefore instructed our observers to maintain fixation at the center of the DVN display while making their aftereffect velocity settings. The observers were questioned throughout each experimental session as to whether they maintained fixation, and trials for which fixation was not maintained were discarded. Eye position was monitored objectively in related experiments (although not for the 3 observers tested here) wherein it was established that the DVN stereophenomenon and its associated MAE could be perceived without eye movements.

Estimates of the position in depth of the recessed moving dotplane relative to the plane of the TV monitor were obtained after all MAE measurements were completed. The observers first adjusted the position of the LED so that it appeared coplanar with the DVN display as viewed binocularly with no attenuating filter. The maximal depth effect was then induced using the 2.5 neutral density filter and the observer adjusted the LED to be coplanar with the farthest dot-plane. Eight settings were made under each of these viewing conditions.

\section{Spatial Properties of the DVN Display}

As noted earlier, the purely central MAE reported here was most consistently produced by a detuned television receiver. The dot profile of the monitor used was approximately Gaussian with an effective width $(2 \sigma)$ of about $1 \mathrm{~mm}$. Thus, the $15^{\circ} \times 11^{\circ}$ field contained about 140 dots horizontally and 103 dots vertically. However, because we lacked information on the statistics of the video noise producing the DVN stimulus, we were unable to directly determine the spatial properties of the DVN display. We chose rather to assess the characteristics of the dot distribution indirectly by determining the degree to which the DVN stimulus affected the visibility of sine-wave gratings of various frequencies (cf. Stromeyer \& Julesz, 1972). This approach has the additional advantage over a direct analysis of video noise of accounting for the optical properties of the monitor as a whole.

A contrast-sensitivity function (CSF) was obtained for each observer under two experimental conditions. In the first condition the beamsplitter of Figure 1 was turned $90^{\circ}$ so that the DVN stimulus and the monitor of an Optronix Model 200 Vision Tester were superimposed. Sine-wave gratings with spatial frequencies of $0.5,1$, $2,4,8,11.4$, and 22.8 cycles per degree (cpd) were presented and a CSF was determined using the method of increasing contrast (cf. Ginsburg \& Cannon, 1983). In the second condition a ground glass screen, back-illuminated to $28 \mathrm{fL}$ using a standard slide projector, replaced the DVN display. A CSF was again determined as described for the first condition.

The difference in the CSFs obtained in the two conditions was taken as a measure of the spatial properties of the DVN stimulus. The difference functions were similar for all 3 observers, and so they have been combined and are shown in Figure 2. The effect of the DVN stimulus was to reduce contrast sensitivity at all spatial frequencies tested above $0.5 \mathrm{cpd}$. The largest reduction occurred at about $8 \mathrm{cpd}$, with the effect diminishing toward lower and higher spatial frequencies.

\section{RESULTS}

When viewing the DVN display with a neutral density filter over one eye, most observers reported the perception of both movement and depth, defined by a distribu- 


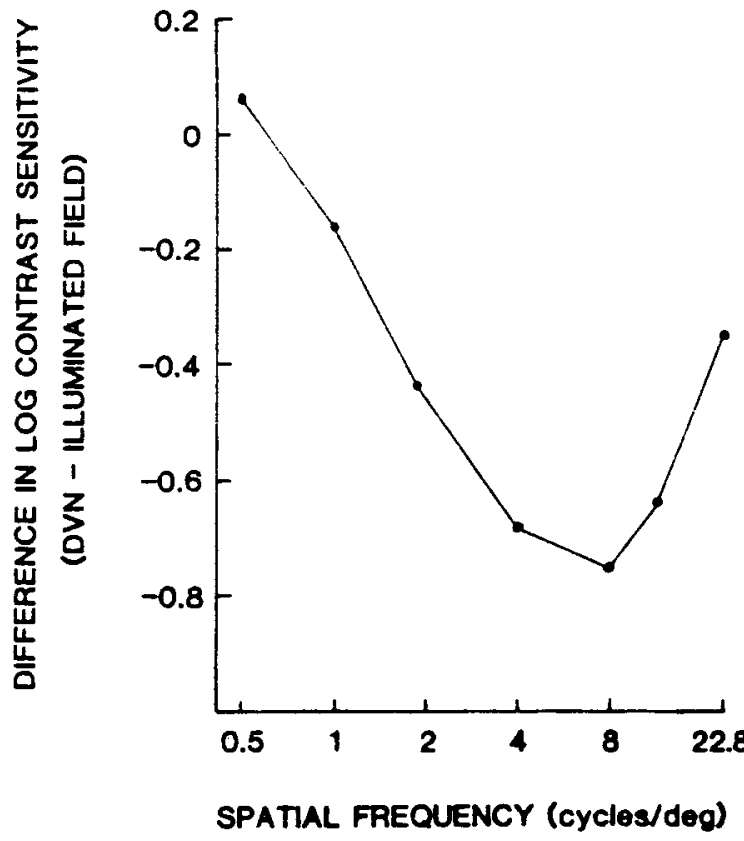

Figure 2. Contrast-sensitivity data obtained to establish the spatial characteristics of the DVN display. On the ordinate is the reduction in log-contrast sensitivity caused by superimposing the DVN stimulus over the sine-wave stimuli of the vision tester.

tion of recessed planes of dots (pixels) moving in the direction of the unfiltered eye and a complementary distribution of protruding planes of dots moving in the opposite direction. In accord with the observations of Falk and Williams (1980), 3 of our observers reported that the farthest dot-plane appeared most distinct. The 3 observers who participated in the present study described the DVN stereophenomenon as consisting of a distinct farthest dotplane, comprising the vast majority of moving dots, with the remaining dots distributed in depth and appearing like "snow flurries." This percept was, in fact, a criterion for selecting the 3 observers.

The solid circles in Figure 3 show, for all 3 observers, data relating the velocity of apparent movement of the dots in the DVN display and the interocular luminance difference. An analysis of variance indicated that dot velocity was an increasing function of the amount of neutral density attenuation placed in front of the right eye $[\mathrm{F}(6,12)$ $=5.50, \mathrm{p}<.01]$.

After viewing the recessed plane for $20 \mathrm{sec}$, all observers saw an MAE when the inducing filter was removed. It appeared as a movement of the DVN field in a direction opposite to that of the inducing (i.e., farthest) dot-plane. The MAE lasted only 1-3 sec, and no perception of depth was associated with it. The duration and magnitude of the MAE did not depend on the time spent viewing the movement associated with the DVN. The open circles of Figure 3 show the relationship between the interocular luminance difference and the velocity of the MAE. The analysis of variance indicated that the MAE was judged faster than the apparent motion which induced it $(\mathrm{p}<.05)$, as can also be observed from the upward shift of most of the MAE data points in Figure 3, and that the velocity of the MAE was independent of the interocular luminance difference $[F(6,12)=1.53, p>.25]$. That is, there was no significant trend in the velocity of the MAE as a function of the interocular luminance difference.

If the velocity of the purely central MAE decayed over time, the MAE would consist of a range of velocities which might preclude an accurate estimation of its velocity by our observers. To compare the variability in MAE velocity estimation with that in induction-field velocity estimation, we summarize in Table 1 the mean and range of standard deviations about the data points of each curve of Figure 3. These data indicate that the observers could estimate aftereffect velocity as accurately as they could estimate the velocity of the movement stimulus. We do not know whether our observers estimated the average or the peak velocity of the MAE, but they were able to do it consistently, and our conclusions are equally valid in either case.

It might be argued that the MAE reported here is due to a difference in the adaptational state of the two eyes at the moment the neutral density filters are removed. In order to obviate this possibility we performed several control studies. ${ }^{1}$

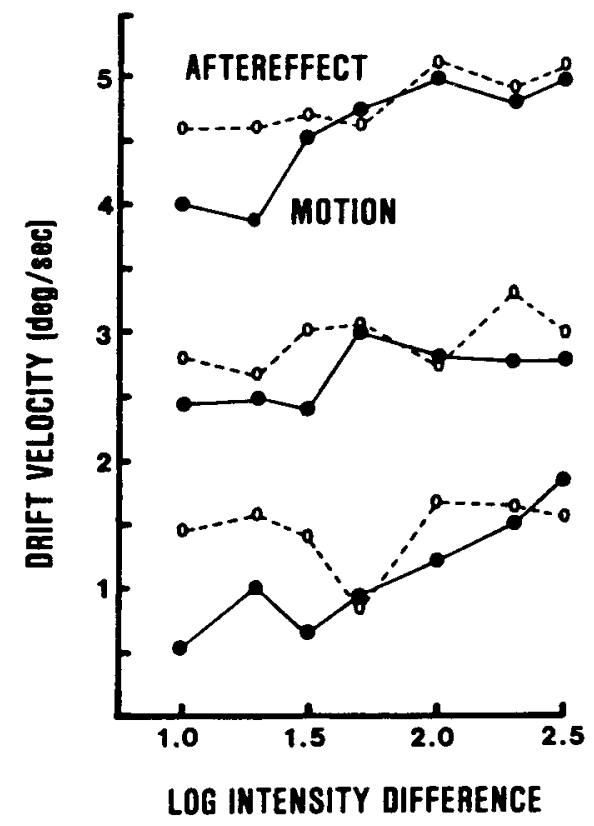

Figure 3. The magnitude of the motion aspect of the DVN stereophenomenon (filled circles) and its associated aftereffect (open circles), both plotted as a function of the interocular intensity difference used to produce the apparent movement stimulus. Although both the movement and its aftereffect increase as a function of the interocular intensity difference, the change in the magnitude of the aftereffect is not statistically significant. The data are from 3 observers, G.G. (top), J.H. (center), and D.W. (bottom). The data shown for G.G. are correctly placed along the ordinate, whereas those for J.H. and D.W. have been shifted downward by $1 \%$ sec and $3 \%$ sec, respectively. Standard deviations are provided in Table 1. 
Table 1

Summary of Data Standard Deviations

\begin{tabular}{clcc}
\hline & & Motion & MAE \\
\hline \multirow{2}{*}{ G.G. } & Mean & 0.56 & 0.62 \\
& Range & $0.44-0.69$ & $0.46-0.78$ \\
J.H. & Mean & 0.48 & 0.65 \\
& Range & $0.34-0.57$ & $0.48-0.79$ \\
D.W. & Mean & 0.53 & 0.70 \\
& Range & $0.46-0.58$ & $0.59-0.78$ \\
\hline
\end{tabular}

\section{DISCUSSION}

There are several notable differences between peripheral or binocular MAEs, produced by stimulus movement on the retina, and what we have called purely central MAEs, produced by cyclopean stimuli which are themselves the result of binocular interaction. Using individual frames composed of Julesz (1960) random-dot stereograms, Papert (1964) produced purely central movement stimuli stereocinematographically. Each eye alone saw a DVN field; the images seen by the two eyes stereoscopically were correlated to produce a bar which appeared to protrude from the surrounding noise and which moved downward. Papert found that such a stimulus produced a central MAE which was shorter for a given inducing time and was more sporadic and less pronounced than peripheral MAEs. Anstis and Moulden (1970) made a further experimental distinction between binocular and purely central (they called them dichoptic) MAEs. Their stimulus consisted of a ring of lights which produced a circular phi-movement, in one direction for each eye viewing it separately and in the opposite direction for the two eyes viewing it together. They, too, noted that the central MAE was of shorter duration and was less pronounced than the monocular MAE. Our observations on the DVN stereophenomenon MAE are consistent with those of Papert and of Anstis and Moulden, and in addition make evident several quantitative differences between purely central MAEs and more conventionally induced MAEs. The data of Figure 3 show that the magnitude of the purely central MAE is independent of the velocity of the inducing stimulus and is greater than that of the movement which produced it.

The distinction drawn above between peripheral or binocular MAEs and purely central MAEs is complicated somewhat ${ }^{2}$ by the observations of Fox et al. (1982) and Stork, Crowell, and Levinson (1985). Fox et al. generated purely central moving contours using the anaglyph technique. The MAEs induced by these contours lasted as long as $\mathbf{2 0 ~ s e c}$ and were reported to be similar to MAEs induced by the movement of physical contours on the retina. Stork et al. (1985) also used moving cyclopean gratings and found that these induced central MAEs which lasted for over $20 \mathrm{sec}$ when no movement existed in the monocular fields used to generate them. These observations are especially difficult to reconcile with those of Papert (1964) and Anstis (1980), who used cyclopean stimuli apparently similar to those used by Fox et al. and Stork et al. However, neither Papert nor Anstis provided sufficient methodological detail to allow us to determine whether quantitative differences in stimulus parameters such as luminance level, dot density, or viewing time may account for the discrepancy between their data and those of the later studies. As to the discrepancy between the present data and those of both Fox et al. and Stork et al., it should be noted that the DVN stimuli used in the latter two studies provided central form or edge information as well as movement information. As discussed, for instance, by Anstis (1980), substantially different MAEs may be expected depending on how associated form and movement mechanisms are stimulated. Thus, at least for the study of purely central MAEs, there appears to be some advantage in using spatially uniform (form-free) DVNinducing stimuli since they are processed exclusively by central movement mechanisms.

Finally, there is another aspect of Fox et al.'s (1982) study that bears on the present results. The duration of the purely central MAE described by Fox et al. was dependent on the relative depth of the inducing and test stimuli. It is possible then that the central MAE described here appeared weak, and hence different from that of Fox et al., because the inducing stimulus was at a different depth plane than the monitor screen which was used to test for the MAE. The present LED depth measurements, however, do not support this interpretation. The difference in apparent depth between the inducing and test stimuli ranged from 7.5 to $10.7 \mathrm{~mm}$. Assuming an interpupillary distance of $60 \mathrm{~mm}$, this depth difference corresponds to a retinal disparity difference of less than 2 arcmin-a value substantially below that determined by Fox et al. to be necessary to affect MAE measurements. Thus, we are left with the conclusion that the form-free characteristics of our stimulus, which are processed by central movement mechanisms, are responsible for the differences between peripheral or binocular MAEs and purely central MAEs, as well as the differences among the purely central MAEs of Fox et al., Stork et al., and the present study.

\section{REFERENCES}

Anstis, S. M. (1980). The perception of apparent movement. Philosophical Transactions of the Royal Society of London, B, 290, 153-168. Anstis, S. M., \& Moulden, B. P. (1970). Aftereffect of seen movement: Evidence for peripheral and central components. Quarterly Journal of Experimental Psychology, 22, 222-229.

BARLOW, H. B., \& BRINDLEY, G. S. (1963). Interocular transfer of movement aftereffects during pressure blinding of the stimulated eye. $\mathrm{Na}$ ture, $200,1347$.

Falk, D. S, \& Williams, R. (1980). Dynamic visual noise and the stereophenomenon: Interocular time delays, depth, and coherent velocities. Perception \& Psychophysics, 28, 19-27.

Favreau, O. E. (1976). Motion aftereffects: Evidence for parallel processing in motion perception. Vision Research, 16, 181-186.

Fox, R., Patterson, R., \& Lehmkuhle, S. (1982). Effect of depth position on the motion aftereffect. Investigative Ophthalmology \& Visual Science, 22(Suppl.), 144.

Ginsburg, A. P., \& Cannon, M. W. (1983). Comparison of three 
methods for rapid determination of threshold contrast sensitivity. Investigative Ophthalmology \& Visual Science, 24, 798-802.

JuLESz, B. (1960). Binocular depth perception of computer-generated patterns. Bell System Technical Journal, 39, 1125-1162.

MACDonALD, R. I. (1977). Temporal stereopsis and dynamic visual noise. Vision Research, 17, 1127-1128.

MEZRICH, J. J., \& Rose, A. (1977). Coherent motion and stereopsis in dynamic visual noise. Vision Research, 17, 903-910.

NeILL, R. A. (1981). Spatio-temporal averaging and the dynamic visual noise stereophenomenon. Vision Research, 21, 673-682.

PAPERT, S. (1964). Stereoscopic synthesis as a technique for localizing visual mechanisms. M.I.T. Quarterly Progress Report No. 73, 239-244.

ROGERS, B. J., \& ANSTIS, S. M. (1972). Intensity versus adaptation and the Pulfrich stereophenomenon. Vision Research, 12, 909-928.

Ross, J. (1974). Stereopsis by binocular delay. Nature, 248, 363-364.

Stork, D. G., Crowell, J. A., \& Levinson, J. Z. (1985). Cyclopean motion aftereffect in the presence of monocular motion. Investigative Ophthalmology \& Visual Science, 26(Suppl.), 55.

STROMEyer, C. F., III, \& JULESZ, B. (1972). Spatial frequency masking in vision: Critical bands and spread of masking. Journal of the Optical Society of America, 62, 1221-1232.

TYLER, C. W. (1974). Stereopsis in dynamic visual noise. Nature, 250, 781-782.

TYLER, C. W. (1977). Stereomovement from interocular delay in dynamic visual noise: A random spatial disparity hypothesis. American Journal of Optometry \& Physiological Optics, 54, 374-386.

WARD, R., \& MoRgan, M. J. (1978). Perceptual effect of pursuit eye movement in the absence of a target. Nature, 274, 158-159.

ZeEvi, Y. Y., \& Medina, A. (1984). Acceleration perceived with dynamic visual noise. Journal of the Optical Society of America, A, 1, 562-564.

\section{NOTE}

1. Several control experiments were performed to establish that the movement perceived in the DVN when the adapting filter was removed was, in fact, an MAE, and was not due to a difference in the adaptational state of the two eyes (see, e.g., Rogers \& Anstis, 1972, for a discussion of the effects of adaptation on the Pulfrich stereophenomenon). A preliminary control for differential light adaptation was performed by dark-adapting one eye for $10 \mathrm{~min}$ while the other eye viewed either the DVN display or even more intense room illumination. These procedures were performed four times each by the 3 observers, and in no case was an MAE seen when the dark-adapted eye was uncovered.

An additional control more nearly duplicated the luminance conditions under which the MAE was originally perceived. The 3 subjects were observer G.G. and 2 observers who saw the DVN MAE but who had not participated in the original study. Two stimulus arrangements were used in an attempt to induce an MAE by differential adaptation. Black-and-white photographs were taken of the DVN display, and they were both printed on photographic paper and made into slide transparencies. Six photographs and six projected transparencies, each representing a contrast series and each with a mean luminance of $28 \mathrm{fL}$, were presented to the observers, who chose the one in each group which appeared most similar to the DVN display. The chosen photograph and transparency as well as the two adjacent to them in the contrast series were used in the control experiments.

The photographs were illuminated by an incandescent lamp and each subject viewed them binocularly for $1 \mathrm{~min}$ with a 2.5 neutral density filter over the right eye. The observer then removed the filter and immediately directed his gaze to the DVN display. The transparencies were back-projected onto a ground glass screen, and a beamsplitter was used to superimpose the transparency and the DVN display. The observer viewed the transparency for $1 \mathrm{~min}$, again with a 2.5 neutral density filter over the right eye. The observer was instructed to remove the filter while the experimenter simultaneously occluded the transparency and exposed the DVN display. This procedure was accomplished manually and took about $1 / 2 \mathrm{sec}$. Despite repeated attempts no observer was able to see an MAE under any of these conditions.

(Manuscript received November 12, 1984; revision accepted for publication October $28,1985$. 